\title{
Enhancing thermally activated delayed fluorescence by fine-tuning the dendron donor strength
}

Eimantas Duda, ${ }^{a}$ David Hall, ${ }^{b, c}$ Sergey Bagnich, ${ }^{a}$ Cameron L. Carpenter-Warren, ${ }^{b}$ Rishabh Saxena, ${ }^{a}$ Michael Y. Wong, ${ }^{b}$ David B. Cordes, ${ }^{b}$ Alexandra M. Z. Slawin, ${ }^{b}$ David Beljonne, ${ }^{c}$ Yoann Olivier, ${ }^{\text {* }}$ Eli Zysman-Colman, ${ }^{b *}$ and Anna Köhler ${ }^{a *}$

aSoft Matter Optoelectronics, BIMF \& BPI, University of Bayreuth, Universitätsstraße 30, 95447 Bayreuth, Germany. E-mail: anna.koehler@uni-bayreuth.de

brganic Semiconductor Centre, EaStCHEM School of Chemistry, University of St Andrews, St Andrews, UK, KY16 9ST

'Laboratory for Chemistry of Novel Materials, University of Mons, 7000, Mons, Belgium dUnité de Chimie Physique Théorique et Structurale \& Laboratoire de Physique du Solide, Namur Institute of Structured Matter, Université de Namur, Rue de Bruxelles, 61, 5000 Namur, Belgium 


\section{Abstract}

Thermally activated delayed fluorescence (TADF) relies on a small energy gap between the emissive singlet and the non-emissive triplet state, obtained by reducing the wavefunction overlap between donor and acceptor moieties. Efficient emission, however, requires maintaining a good oscillator strength, which is itself based on sufficient overlap of the wavefunctions between donor and acceptor moieties. We demonstrate an approach to subtly fine-tune the required wavefunction overlap by employing donor-dendrons of changing functionality. We use a carbazolyl-phthalonitrile based donoracceptor core, $\mathbf{2 C z P N}$, as a reference emitter, and progressively localize the hole density through substitution at the 3,6-positions of the carbazole donors $(\mathbf{C z})$ with further carbazole, (4-tertbutylphenyl)amine ('BuDPA) and phenoxazine (PXZ). Using detailed photoluminescence studies, complemented with Density Functional Theory (DFT) calculations, we show that this approach permits a gradual decrease of the singlet-triplet gap, $\Delta E_{S T}$, from $300 \mathrm{meV}$ to around $10 \mathrm{meV}$ in toluene, yet we also demonstrate why a small $\Delta E_{S T}$ alone is not enough. While sufficient oscillator strength is maintained with the Cz- and ${ }^{t} \mathrm{BuDPA}$-based donor dendrons, this is not the case for the PXZ-based donor dendron, where the wavefunction overlap is reduced too strongly. Overall, we find the donordendron extension approach allows successful fine-tuning of the emitter photoluminescence properties. 


\section{Introduction}

Emitters showing thermally activated delayed fluorescence (TADF) can achieve comparable internal quantum efficiencies to those of commercialised phosphorescent emitters, but without the need to employ scarce heavy metals within an organic light emitting diode (OLED) $\cdot{ }^{1-3}$ TADF allows non-coherent endothermic triplet exciton upconversion to the singlet state, mediated by the small singlet-triplet splitting. This is particularly useful when employing these materials for OLED applications, since in this way, all electrically generated excitons in the OLED may be used towards light emission. The energy gap between the lowest singlet and triplet states, $\Delta E_{S T}$, that governs in part the efficiency of the TADF process depends on the magnitude of the wavefunction overlap in the exchange integral. ${ }^{4-5}$ The singlettriplet gap can therefore be reduced by localizing hole and electron wavefunctions onto different parts of the molecule, and this is commonly achieved by employing, respectively, donor and acceptor moieties that adopt a highly twisted conformation in the emitter structure. Nevertheless, there needs to be sufficient wavefunction overlap to still allow for a reasonable transition dipole moment, and thus a good radiative decay rate. ${ }^{4-5}$ The balance of these two factors is required to obtain a highperformance TADF emitter.

One way to adjust wavefunction overlap is by modulating the torsion between the donor and the acceptor moieties, which controls the extent of the conjugation between these two fragments. ${ }^{6-9} \mathrm{An}$ alternative way is to increase the delocalization of the hole over the donor unit, which effectively localizes the hole density on this fragment. ${ }^{10}$ Implementation of this strategy is illustrated in dendrimers, which frequently contain dendritic donors surrounding an electron-acceptor. ${ }^{5,11-14}$ It has been shown that the more moderate D-A torsions in the dendrimers would ensure sufficient overlap between HOMO and LUMO wavefunctions to achieve the paradox of a small $\Delta E_{S T}$ and a large oscillator strength. ${ }^{7}$ A further advantage of employing a dendritic structure for the emitter is to potentially obviate the need for a host matrix in the emitter layer of an OLED. ${ }^{2,15}$ Commonly, TADF emitters are dispersed in a host matrix to reduce concentration quenching. The dendritic structure itself is often 
sufficiently sterically crowded to mitigate concentration quenching of the vulnerable triplet states. Concentration quenching can be synergistically further suppressed by the use of stericly bulky groups decorating the donor dendrons. This facilitates the device fabrication and avoids issues associated with host-guest interaction such as exciplex formation and undesirable energy shifts. ${ }^{16}$ There are, however, few variants of donor dendrons that have been explored. An appreciation of the effect of structure modulation on the optoelectronic properties, particularly on the efficiency of the TADF process, is thus currently missing. A toolbox-like approach is desirable, where different donor units can be connected with ease to gently and gradually modify the properties. ${ }^{17}$

Here we demonstrate such a modular fine-tuning approach by changing the nature of the peripheral donor groups in donor dendrons, which affects hole localization, while maintaining the central carbazole donor unit and its connection to the common phthalonitrile acceptor. This strategy avoids re-optimizing the entire molecular design. We are not aware of detailed and systematic studies on the impact of the nature of the donor dendrons on the singlet-triplet gap and TADF properties. An overview of literature-reported emitters containing donor dendrons, including dendrimers, is given in the ESI (Table S1, Figure S1). We address whether such a strategy can significantly improve the efficiency of the TADF process in an emitter that already shows TADF in thin films.

We implement our approach by changing the peripheral donor groups on the TADF-active sky-blue emitter 2CzPN (Figure 1). ${ }^{18-20}$ 2CzPN consists of an electron-accepting phthalonitrile core connected to two carbazole donor moieties at the 4- and 5-positions. These donors can be substituted comparatively easily at the 3,6-positions of the carbazole, allowing for the extension of the conjugated $\pi$-system. The compound is thus well suited to study the impact of hole wavefunction delocalization on the singlettriplet gap and the TADF performance. $2 \mathrm{CZ} \mathbf{P N}$ shows a desirable set of photophysical properties in terms of its high reported photoluminescence quantum yield (PLQY) of $89 \%$ and the reported presence of TADF in an mCP matrix, ${ }^{19-20}$ although we find a surprisingly high $\Delta E_{\mathrm{ST}}$ gap of $230 \mathrm{meV}$ in our 
measurements in a film with $\mathrm{mCP}$ as host matrix, increasing to $300 \mathrm{meV}$ in toluene solution. To gradually reduce electron-hole overlap we progressively altered the electronic structure of the donor dendron, by decorating the central carbazole with either peripheral carbazole $(\mathbf{C z})$, di(4-tertbutylphenyl)amine ('BuDPA) or phenoxazine (PXZ) groups.

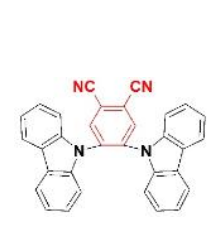

2CzPN

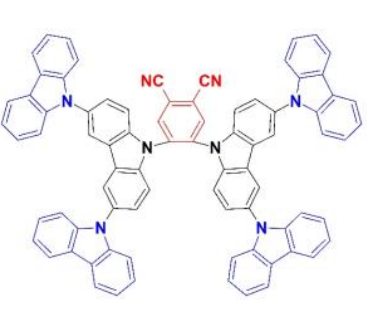

$\mathrm{Cz}-2 \mathrm{CzPN}$

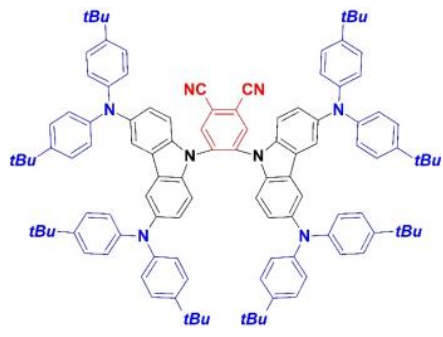

${ }^{t}$ BuDPA-2CzPN

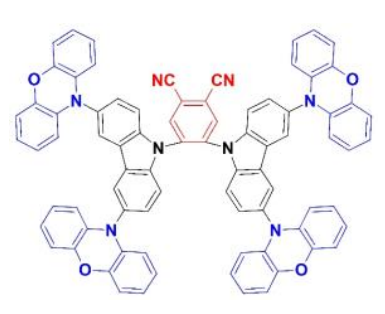

PXZ-2CzPN

Figure 1. Chemical structures of the investigated compounds. The parent $2 \mathrm{Cz} \mathbf{P N}$ is extended using carbazole (Cz), di(4-tert-butylphenyl)amine( ${ }^{t}$ BuDPA) and phenoxazine (PXZ).

We find that our approach allows for an enhancement of the intramolecular charge transfer (CT) character of the emitter and a progressively smaller $\Delta E_{S T}$, from $300 \mathrm{meV}$ for the parent compound 2CzPN to $160 \mathrm{meV}$ for Cz-2CzPN and $10 \mathrm{meV}$ for the other two compounds in toluene. Consistent with this, the optical spectroscopy as well as density functional theory (DFT) calculations suggest that the overlap of electron and hole density reduces along the series, until for PXZ-2CZPN, the hole density is localized only on the PXZ units, thus limiting the overlap with the electron density localized on the phthalonitrile unit. We also find that non-radiative losses are present in the case of $\mathbf{C z}-\mathbf{2} \mathbf{C z P N}$ and ${ }^{t}$ BuDPA-2CzPN, supressing the full TADF potential. Nevertheless, overall, we find the donor-dendron extension approach allows for the optimization of the hole delocalization and thus, for $\mathbf{C z}-\mathbf{2} \mathbf{C z P N}$, to show an increase in PLQY over the parent compound. This improvement in $\Delta E_{S T}$ comes, however, at the expense of a redshift in the emission colour. 


\section{Theoretical calculations}

DFT calculations were undertaken to elucidate the effect of changing the donor in the $2 \mathrm{CzPN}$ derivatives on the distribution of electron and hole densities, the magnitude of the oscillator strengths for the vertical transition between the ground state and the singlet excited states, and the orbital and state energies. Figure $\mathbf{2}$ presents the hole and electron densities for the lowest-lying excited singlet state calculated within the attachment/detachment formalism and the orbital and state energies from the ground state geometry. ${ }^{21}$ The lowest-lying triplet state electron and hole densities can be found in the ESI (Figure S2). ${ }^{22}$ This approach provides information about the reorganization of the electron density upon excitation of the molecule.

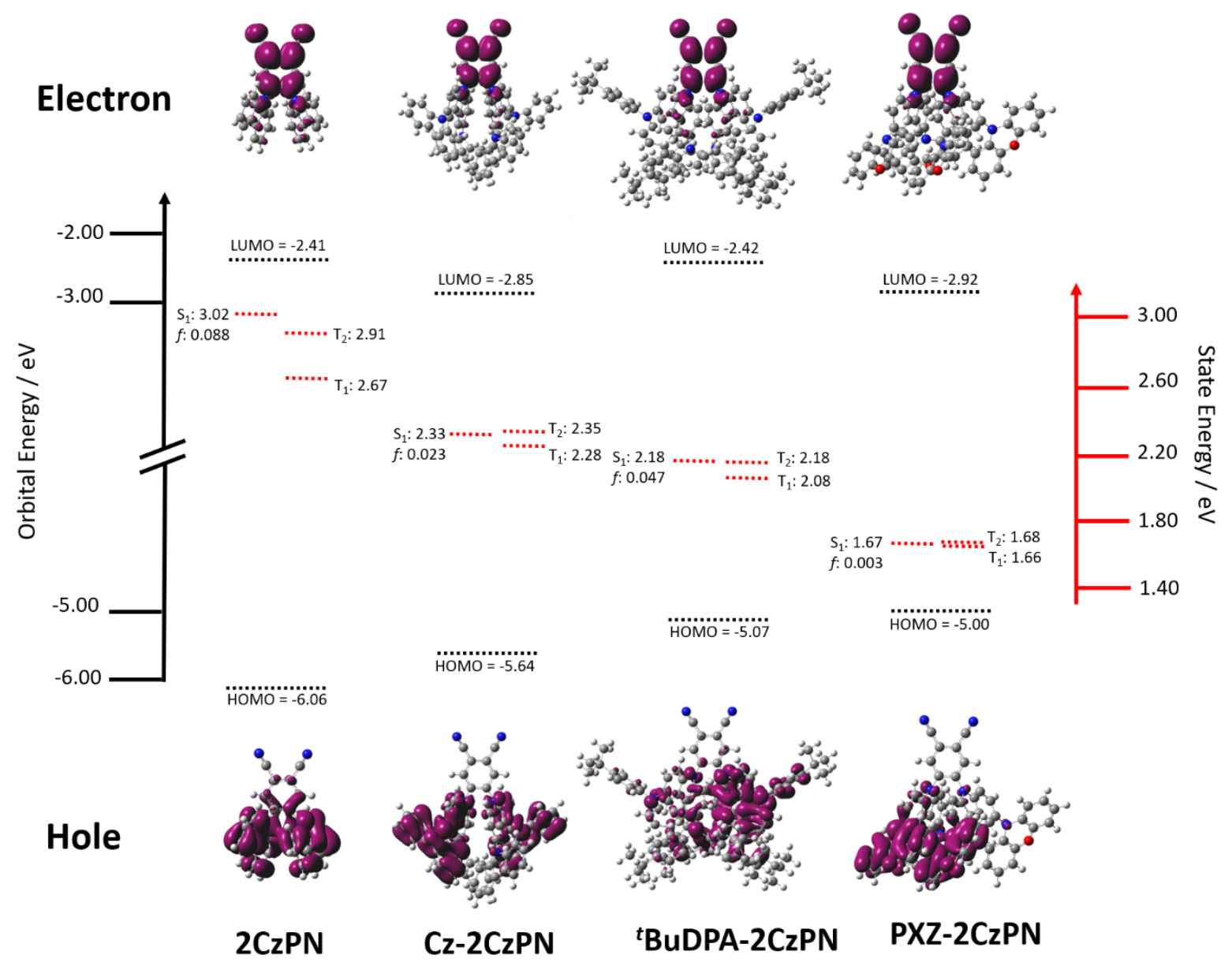

Figure 2. Left axis (black): HOMO and LUMO energies calculated for the ground state geometry at the PBE0/6-31G(d,p) level. Right axis (red): vertical transition energies for $S_{1}, T_{1}$ and $T_{2}$ computed at the 
TDA-DFT using the PBE0 functional together with the 6-31G(d,p) basis set. Also shown are the electron (top) and hole densities (bottom) for the for $\mathrm{S}_{1}$ state, where $f$ is the oscillator strength.

For all compounds, the electron density is localized on the phthalonitrile acceptor. In contrast, the localization of the hole density varies across the different emitters. For $\mathbf{2} \mathbf{C z} \mathbf{P N}$, the hole density is mainly localized on the carbazole donors with some delocalization onto the phthalonitrile phenyl ring. When extending the donor as in Cz-2CzPN and ${ }^{t}$ BuDPA-2CzPN, however, the hole density is distributed across the now larger donor dendron moieties, with a reduced contribution on the phthalonitrile unit. For PXZ-2CZPN the hole density is mostly localized on the distal phenoxazine donors, with only a slight contribution from the inner carbazole. Close inspection reveals that the hole density on the phthalonitrile accepting unit and the carbon atoms connected to it decreases when moving from ${ }^{t}$ BuDPA-2CzPN to Cz-2CzPN and PXZ-2CzPN. As discussed further below, this related to changes in donor strength and the donor-acceptor torsion. The clear separation between electron and hole densities in each compound indicates that the singlet excited state possesses a dominant chargetransfer (CT) character. The degree of CT character can be quantified using the parameter $\varphi_{\mathrm{S}}$, where a value of 1 is indicative of a state of purely locally excited (LE) character and a value of 0 is a state of purely $\mathrm{CT}$ character with the former signifying complete overlap and the latter no overlap. The $\varphi_{\mathrm{s}}$ value decreased from 0.42 for 2 CzPN to $0.29,0.20$ and 0.11 for ${ }^{t}$ BuDPA-2CzPN, Cz-2CzPN and PXZ-2CzPN, respectively, for the $S_{1}$ state suggesting an increase in $\mathrm{CT}$ character and supporting the decrease of the oscillator strength, $f$ (see Figure 2 ). The electron and hole densities for the first triplet excited state closely resemble those of the $S_{1}$ state and are given in the ESI (Figure S2). For each compound, we also calculated the energy of the $T_{2}$ state and characterized its nature. The presence of intermediate triplet states energetically close to the $T_{1}$ and $S_{1}$ states is essential for efficient TADF to take place. Indeed, El Sayed's rules essentially forbid the direct $T_{1}$ and $S_{1}$ conversion in cases where the states have the same natures, as would be the case when both are pure CT states, due to the predicted vanishing spin-orbit coupling. We consider that for our compounds, the upconversion process from $T_{1}$ to $S_{1}$ involves reverse 
internal conversion from $T_{1}$ to $T_{2}$ driven by vibronic coupling, followed by efficient Reverse Intersystem Crossing (RISC) to $S_{1}$ in the case where $T_{2}$ is of a different nature than $S_{1}$. Such a mechanism supports the high $k_{\text {RISC }}$ rates observed even in the case of $\Delta \mathrm{E}_{S T}$ values exceeding $200 \mathrm{meV}$. ${ }^{23-24}$ For $2 \mathrm{CZ} \mathrm{PN}$, the $\mathrm{T}_{2}$ state lies between the $T_{1}$ and $S_{1}$, while in each of the other three compounds it is either the same energy or slightly higher in energy than that of the $\mathrm{S}_{1}$ state. Owing to the similar energy levels of $\mathrm{S}_{1}$ and $T_{2}$ it is likely that these states exhibit non-vanishing intersystem crossing. Further, $T_{1}$ and $T_{2}$ are energetically close and could show efficient vibronic coupling.

The calculated HOMO energy progressively destabilizes, indicative of both the increasing strength of the donors as well as the delocalization of the HOMO orbital, across the series (Table 1). An unexpected trend is observed with the calculated LUMO energy. Both 2 CzPN and ${ }^{t}$ BuDPA-2CzPN have similar energies at $-2.41 \mathrm{eV}$ and $-2.42 \mathrm{eV}$, respectively. By contrast, a significant stabilisation is observed for Cz-2CzPN and PXZ-2CZPN, with LUMO energies of $-2.85 \mathrm{eV}$ and $-2.92 \mathrm{eV}$, respectively. The change in behaviour is likely related to the calculated torsional angles between the carbazole donor and phthalonitrile acceptor, with the torsions being greatest for PXZ-2CzPN and Cz-2CzPN, with average angles between the respective planes of $60^{\circ}$ and $67-70^{\circ}$, respectively, compared to $53^{\circ}$ and $56^{\circ}$ for both 2 CzPN and ${ }^{t}$ BuDPA-2CzPN. The smaller torsion in the latter ensures a larger delocalization of the LUMO orbital onto the electron-donating $\mathrm{Cz}$ groups. These trends were also observed by $\mathrm{X}$-ray crystallography (vide infra). The HOMO-LUMO gap is strongly affected by the changes in HOMO and LUMO energies, with values ranging from $2.08 \mathrm{eV}$ for PXZ-2CZPN to $3.65 \mathrm{eV}$ for $2 \mathrm{CZPN}$. The trend in the HOMO-LUMO gap (Table 1) aligns also with the trend observed for the $\mathrm{S}_{1}$ energies (Table 2) because this excited state is mainly described (> 90\%) by a HOMO to LUMO transition. The increasing $\mathrm{CT}$ character along the series is associated with a smaller calculated $\Delta E_{S T}$, with the values decreasing from $0.34 \mathrm{eV}$ in 2CZPN to $0.01 \mathrm{eV}$ in PXZ-2CzPN (Table S2). This is consistent with the values measured by photoluminescence spectroscopy (Table 3). When M062X is employed as the functional in lieu of PBEO in the DFT calculations, identical trends are observed; however, the magnitude of the CT 
character changes slightly, owing to the changing HF content ( $56 \%^{25}$ for M062X and $25 \%^{26}$ for PBEO). This increased HF contribution helps to alleviate overstabilisation of the CT states; ${ }^{27}$ however, as the $\mathrm{S}_{1}$ and $\mathrm{T}_{1}$ states are $\mathrm{CT}$ in nature in these compounds, the differences between the two methods are minimal. Data from the M062X calculations, including oscillator strength, $\varphi_{\mathrm{S}}, \Delta E_{\mathrm{ST}}$, calculated $\mathrm{HOMO}$ LUMO energies, can be found in the ESI (Table S3). Overall, the DFT data suggest that the design concept of reducing the singlet-triplet gap by increasing the strength of the peripheral donors of the donor dendrons is reasonable.

\section{Synthesis, chemical and electrochemical characterisation}

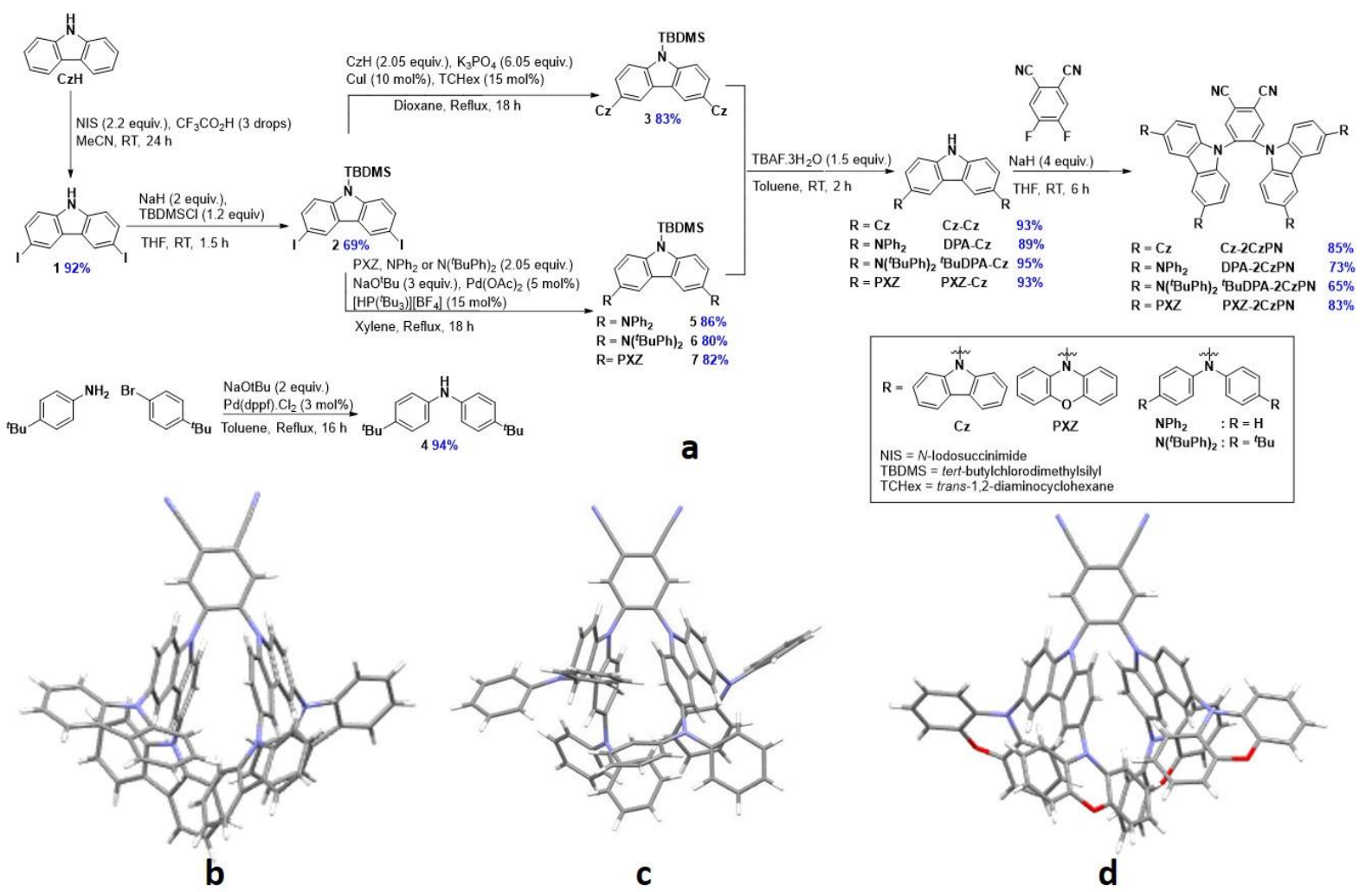

Figure 3. (a) Synthesis of the target emitters and single crystal structures of (b) Cz-2CzPN, (c) DPA-

2CZPN and (d) PXZ-2CzPN. 
The synthesis of the emitters is outlined in Figure 3a. The identity and purity of all compounds were determined from a combination of NMR spectroscopy, high resolution mass spectrometry, elemental analysis and melting point determination. In addition, single crystals of Cz-2CzPN, DPA-2CzPN and PXZ2CZPN were each obtained either from the layering of a saturated solution of toluene with hexane $(\mathbf{C z}-$ 2CzPN, PXZ-2CzPN) or from the slow evaporation of a toluene solution (DPA-2CzPN, PXZ-2CzPN). Two structures of PXZ-2CZPN, showing different solvation and slightly different conformations, were obtained, designated PXZ-2CZPN(A) and PXZ-2CZPN(B). Owing to the insolubility of DPA-2CzPN, ${ }^{t}$ BuDPA-2CzPN was also synthesised and we hypothesise that the addition of ${ }^{t} \mathrm{Bu}$ groups should have a minimal impact on the photophysics while addressing the issues of solubility. This is corroborated by DFT calculations that predict nearly identical $\mathrm{S}_{1}\left(2.19 \mathrm{eV}\right.$ and $2.18 \mathrm{eV}$ for DPA-2CzPN and ${ }^{\text {B BuDPA- }}$ 2CZPN, respectively) and $\Delta E_{S T}$ (0.10 for both) values for the two compounds (Figure $\mathbf{S 3}$, Tables $\mathbf{S 2}$ and S3). No further photophysical analysis was undertaken for DPA-2CzPN owing to its insolubility but we discuss its crystal structure as crystals of ${ }^{t}$ BuDPA-2CzPN were not obtained and the conformations are calculated to be similar (Table S5).

Cz-2CzPN, DPA-2CzPN and PXZ-2CzPN display generally similar torsion angles between the inner carbazole donor and the adjacent phthalonitrile acceptor, although greater variation is seen both between the two forms of PXZ-2CZPN and also between the torsion angles in PXZ-2CZPN(A) [average torsion for each carbazole Cz-2CzPN 61.5 and 63.1 ${ }^{\circ}$, DPA-2CzPN 54.5 and 59.1 ${ }^{\circ}$, PXZ-2CzPN(A) 49.4 and $57.3^{\circ}$, and PXZ-2CzPN(B) 60.0 and $65.0^{\circ}$. The DFT-calculated inner dihedral angles, $53.8^{\circ}$ (2CzPN), 60.2 and $60.3^{\circ}\left(\mathrm{CZ}^{2}-2 \mathrm{Cz} P N\right), 53.4^{\circ}$ (DPA-2CzPN), 66.5 and $70.2^{\circ}$ (PXZ-2CzPN), agree well with those from the crystal structures. The torsions calculated from DFT and measured from the crystal structures are summarized in Table S5. The differences between the two structures of PXZ-2CZPN include differences in torsions between rings, such as those mentioned above, as well as differences in the extent of puckering of the PXZ groups (Figure S4). The PXZ groups adopt varying degrees of puckered conformation between the two structures, similar to that observed previously in structurally similar 
phenothiazine-containing compounds. ${ }^{28}$ In PXZ-2CZPN(A) two PXZ groups are near-planar (PXZ pucker angles of 1.4 and $4.9^{\circ}$ ), while two adopt a moderate pucker (angles of 13.8 and $17.3^{\circ}$ ) whereas in PXZ2CzPN(B) two PXZ groups show slight pucker (angles of 8.7 and $10.0^{\circ}$ ) while the other two show a greater pucker than any other PXZ in either structure (angles of 20.6 and $23.6^{\circ}$ ). The compounds have D-A torsions generally larger than those reported for 2 CzPN, which ranged between 49.3 and $59.8^{\circ}$, and which can be attributed to the increased steric bulk of the dendrons. The donor-donor torsions vary within individual compounds but show broad trends across the series. For $\mathbf{C z}-\mathbf{2 C z P N}$, the torsions of the peripheral carbazoles to the central carbazole range between 59.0 and $78.5^{\circ}$. Due to the larger size of the PXZ groups, there is a slightly larger set of PXZ-carbazole torsions [PXZ-2CZPN(A) 70.0-78.6 ${ }^{\circ}$, PXZ-2CzPN(B) $70.6-79.2^{\circ}$ ] observed in the two structures. Smaller torsions $\left(44.3-65.8^{\circ}\right)$ were observed between the DPA groups and the central carbazole in DPA-2CzPN. These smaller dihedral angles should result in an increased conjugation across the dendron, increasing the effective donor strength (vide infra).

To experimentally determine the HOMO and LUMO levels, cyclic voltammetry (CV) and differential pulsed voltammetry (DPV) were performed on each of the four emitters as well as on the isolated donor groups (and partial donor groups) in dichloromethane (DCM) (Figure 4 and Figures S5-S9). A summary of the electrochemical data is found in Table $\mathbf{1}$ for the emitters, and in the ESI (Table S6) for the donor groups. 


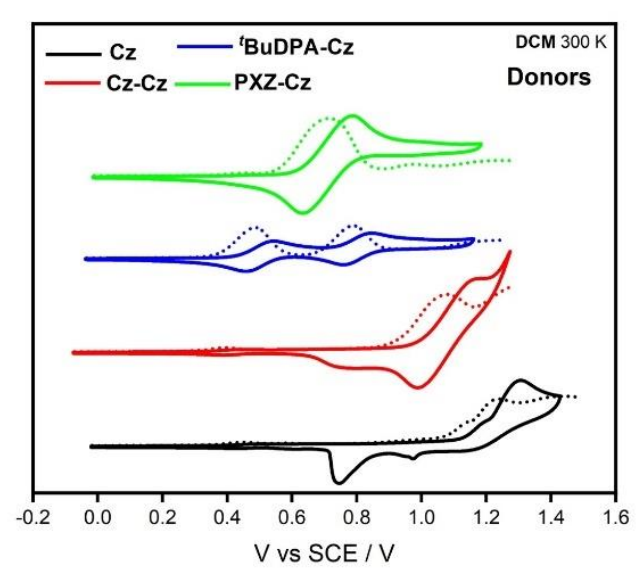

a

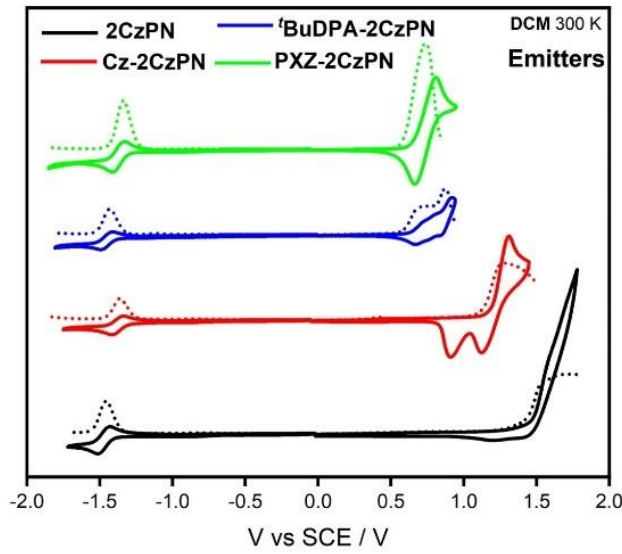

b

Figure 4. Cyclic voltammetry (solid trace) and differential pulsed voltammetry (dashed trace) in DCM of (a) the isolated donor groups; (b) the emitters.

We first investigated the electrochemical behaviour of the isolated donor groups in DCM. As expected, the oxidation of carbazole is not reversible and the compound degrades upon successive scans (Figure S6), via a putative electropolymerisation. ${ }^{29}$ The $E^{\text {ox }}$ from the DPV of $1.15 \mathrm{~V}$ versus SCE matches that previously reported ( $\mathrm{E}^{\mathrm{ox}}=1.16 \mathrm{~V}$ in $\mathrm{MeCN}$ ). ${ }^{30}$ Both ${ }^{\mathrm{t}} \mathrm{BuDPA}$ and PXZ display quasi-reversible oxidation waves centred at $0.84 \mathrm{~V}$ and $0.66 \mathrm{~V}$ (Figure S5), respectively, indicating that PXZ is the strongest donor. The $\mathrm{E}^{\mathrm{ox}}$ of $\mathrm{Cz}-\mathrm{Cz}$ is cathodically shifted with respect to $\mathrm{Cz}$ at $1.08 \mathrm{~V}$, in agreement with a similar derivative (wherein the $\mathrm{N}-\mathrm{H}$ of $\mathrm{Cz}-\mathrm{Cz}$ was replaced by $\mathrm{N}-\mathrm{C}_{6} \mathrm{H}_{13}$ ) with $\mathrm{E}^{\text {ox }}$ also at $1.08 \mathrm{~V}$ in DCM. ${ }^{29,31}$ Only a single oxidation wave is observed, suggesting that the radical cation is delocalized over the $\mathrm{Cz}-\mathrm{Cz}$ molecule. $\mathrm{Cz}-\mathrm{Cz}$ is electrochemically unstable, as evidenced by the evolution of the CVs over multiple scans where a second oxidation wave appears (Figure S6), attributed to polymerisation of the carbazole species. For ${ }^{t}$ BuDPA-Cz there are two observed reversible oxidation waves (Figure S7) assigned to the oxidation of carbazole and then ${ }^{t}$ BuDPA (Figure 4a). These are both cathodically shifted compared those of $\mathrm{Cz}(1.16 \mathrm{~V})$ and ${ }^{t} \mathrm{BuDPA}(0.84 \mathrm{~V})$, the $\mathrm{E}^{\mathrm{ox}}$ of the first wave is $0.49 \mathrm{~V}$. For PXZ-Cz, there is a single reversible oxidation wave at $0.71 \mathrm{~V}$ assigned to oxidation of $\mathrm{PXZ}$ ( $\mathrm{E}^{\mathrm{ox}}$ of $\mathrm{PXZ}=0.66 \mathrm{~V}$, Figure S7). Although PXZ is a stronger donor than ${ }^{t}$ BuDPA based on its cathodically shifted oxidation 
potential, the ability for ${ }^{t} \mathrm{BuDPA}$ to effectively conjugate to carbazole ensures that ${ }^{t} \mathrm{BuDPA}-\mathrm{Cz}$ ( $\mathrm{E}^{\text {ox }}$ of $0.49 \mathrm{~V})$ is a stronger donor than PXZ-CZ ( $E^{\text {ox }}$ of $\left.0.71 \mathrm{~V}\right)$.

We next investigated to electrochemistry of the emitters. The CV of 2CzPN shows an irreversible oxidation wave (Figure S5) with $\mathrm{E}^{\mathrm{ox}}$ at $1.54 \mathrm{~V}$. The calculated $\mathrm{HOMO}$ at $-5.87 \mathrm{eV}$ reproduces that previously reported $\left(\mathrm{E}_{\text {номо }}=-5.88 \mathrm{eV}\right) .{ }^{20}$ The irreversible oxidation wave (Figure $\mathbf{S 5}$ ) for $\mathbf{C z}-\mathbf{2 C z P N}$ is cathodically shifted at $\mathrm{E}^{\mathrm{ox}}$ of $1.28 \mathrm{~V}$ due to the stronger donor. There are two observed oxidation processes in the DPV for ${ }^{\text {t}}$ BuDPA-2CzPN (Figure $\mathbf{4 b}$ ). The first oxidation peak potential is significantly cathodically shifted at $0.72 \mathrm{~V}$ compared to that of $\mathbf{C z - 2 C z P N}$, reflecting the much stronger donor character of the ${ }^{t} \mathrm{BuDPA}-\mathrm{Cz}$ donor dendron. The $\mathrm{E}^{\text {ox }}$ for ${ }^{t} \mathrm{BuDPA}-\mathbf{2 C z P N}$ is anodically shifted compared to ${ }^{t} \mathrm{BuDPA}-\mathrm{Cz}$ due to the conjugation with the electron-accepting phthalonitrile. The reversible oxidation (Figure S7) of PXZ-2CZPN occurs almost exclusively on the PXZ moieties, with an Ex of $0.80 \mathrm{~V}$, which is slightly anodically shifted compared to the oxidation potential of PXZ-CZ (E of $0.71 \mathrm{~V}$ ). This assignment is supported by DFT calculations that reveal that the HOMO density resides on the PXZ units (Figure 2). The increasing donor strength is largely reflected in the oxidization potentials, with $\mathrm{E}^{\text {ox }}$ of 1.54 V, 1.28 V, 0.72 V and 0.80 V for 2CzPN, Cz-2CzPN, ${ }^{t}$ BuDPA-2CzPN and PXZ-2CzPN, respectively. The relative order of the oxidation potentials reflects the interplay between the degree of conjugation between the peripheral donors and the central carbazole and the strength of the peripheral donors.

Reversible reduction waves are observed for all four emitters, assigned to the reduction of the phthalonitrile acceptor (Figure S9). The reduction potentials are more catholically shifted for $\mathbf{2 C Z P N}$ and ${ }^{t}$ BuDPA-2CzPN with $\mathrm{E}^{\text {red }}$ of $-1.46 \mathrm{~V}$ and $-1.43 \mathrm{~V}$, compared to those of $\mathrm{Cz}-2 \mathrm{CzPN}$ and PXZ-2CzPN ( $\mathrm{E}^{\text {red }}$ of $-1.36 \mathrm{~V}$ and $-1.30 \mathrm{~V}$, respectively, Figure $\mathbf{S 9}$ ). This trend is corroborated by DFT calculations (Figure 2). We attribute these trends to an increased electronic interaction between the donors and the acceptor in $\mathbf{2 C z} \mathbf{P N}$ and ${ }^{\mathrm{t}} \mathrm{BuDPA}-\mathbf{2 C z} \mathbf{P N}$ owing to the less twisted conformation present in these two compounds. Increased electron density on the electron-accepting unit destabilizes the LUMO with 
respect to the more twisted conformers. Indeed, the calculated D-A torsions of $54^{\circ}$ for both compounds, with associated larger $\varphi_{\mathrm{S}}$ values of 0.34 and 0.28 , respectively, indicate greater LE character in these two compounds whereas in $\mathbf{C z - 2 C z P N}$ and PXZ-2CzPN the larger respective D-A torsions of $60^{\circ}$ and $67-70^{\circ}$ are linked with $\varphi_{\mathrm{S}}$ of 0.20 and 0.11 , pointing to greater $\mathrm{CT}$ character. Overall, there is good agreement between experimentally determined and calculated HOMO and LUMO values. The HOMO-LUMO gaps are $3.0 \mathrm{eV}, 2.6 \mathrm{eV}, 2.1 \mathrm{eV}$ and $2.1 \mathrm{eV}$ for $2 \mathrm{CZPN}, \mathrm{Cz}-2 \mathrm{Cz}$ PN, ${ }^{\mathrm{t} B u D P A}-2 \mathrm{Cz} P N$ and PXZ-2CzPN, respectively. Although ${ }^{t}$ BuDPA-2CzPN and PXZ-2CzPN have differing conjugation lengths, and donor strengths, they nevertheless show similar $\Delta E$.

Table 1. Electrochemical data.

\begin{tabular}{|c|c|c|c|c|c|c|c|c|}
\hline Compound & $\begin{array}{l}E^{o x(a)} \\
\text { (V) }\end{array}$ & $\begin{array}{l}E^{\text {red (a) }} \\
\text { (V) }\end{array}$ & $\begin{array}{c}\text { HOMO }^{(b)} \\
(e V)\end{array}$ & $\begin{array}{c}\text { LUMO }^{(b)} \\
(\mathrm{eV})\end{array}$ & $\begin{array}{c}\Delta \mathrm{E}_{\mathrm{H}-\mathrm{L}}{ }^{(\mathrm{c})} \\
(\mathrm{eV})\end{array}$ & $\begin{array}{l}\text { HOMO } \\
\text { (eV) }\end{array}$ & $\begin{array}{l}\text { LUMO } \\
\text { (eV) }\end{array}$ & $\begin{array}{l}\Delta \mathrm{E}_{\mathrm{H}-\mathrm{L}} \\
(\mathrm{eV})\end{array}$ \\
\hline $2 \mathrm{CzPN}$ & 1.54 & -1.46 & -5.87 & -2.88 & 2.99 & -6.06 & -2.41 & 3.65 \\
\hline $\mathrm{Cz}-2 \mathrm{CzPN}$ & 1.28 & -1.36 & -5.62 & -2.98 & 2.64 & -5.63 & -2.85 & 2.78 \\
\hline${ }^{t} \mathrm{BuDPA}-2 \mathrm{Cz} \mathrm{PN}$ & 0.72 & -1.43 & -5.04 & -2.92 & 2.12 & -5.07 & -2.42 & 2.65 \\
\hline PXZ-2CzPN & 0.80 & -1.30 & -5.14 & -3.03 & 2.11 & -5.00 & -2.92 & 2.08 \\
\hline \multirow{2}{*}{\multicolumn{9}{|c|}{$\begin{array}{l}\text { (a) Reported versus SCE in degassed DCM with } 0.1 \mathrm{M}\left[n \mathrm{Bu}_{4} \mathrm{~N}\right] \mathrm{PF}_{6} \text { as the supporting electrolyte and } \mathrm{Fc} / \mathrm{Fc}^{+} \\
\text {as the internal reference }(0.46 \mathrm{~V} \text { vs. SCE })^{32} \text { calculated from DPV. } \\
\text { (b) The HOMO and LUMO energies were determined using the relation } \mathrm{E}_{\text {Hомо }} / \mathrm{LUMO}=-\left(\mathrm{E}^{\text {ox }} / \mathrm{E}^{\text {red }}+4.8\right)\end{array}$}} \\
\hline & & & & & & & & \\
\hline \multirow{2}{*}{\multicolumn{9}{|c|}{$\mathrm{eV}^{33}$ where $\mathrm{E}^{\mathrm{ox}}$ and $\mathrm{E}^{\mathrm{red}}$ are the anodic and cathodic peak potentials, respectively measured from DPV }} \\
\hline & & & & & & & & \\
\hline${ }^{2} \mathrm{E}_{\mathrm{H}-\mathrm{L}}=\mid \mathrm{E}_{\text {номо }}-\mathrm{E}$ & & & & & & & & \\
\hline
\end{tabular}




\section{Photophysics in solution and film}

The photophysical data is collated in Table 2. We first consider the compounds in dilute toluene solution ( $0.05 \mathrm{mg} / \mathrm{mL}$, corresponding to $10^{-4}-10^{-5} \mathrm{M}$, depending on compound) where intermolecular interactions can be expected to be supressed. This should provide a clear photophysical understanding related to monomolecular processes.

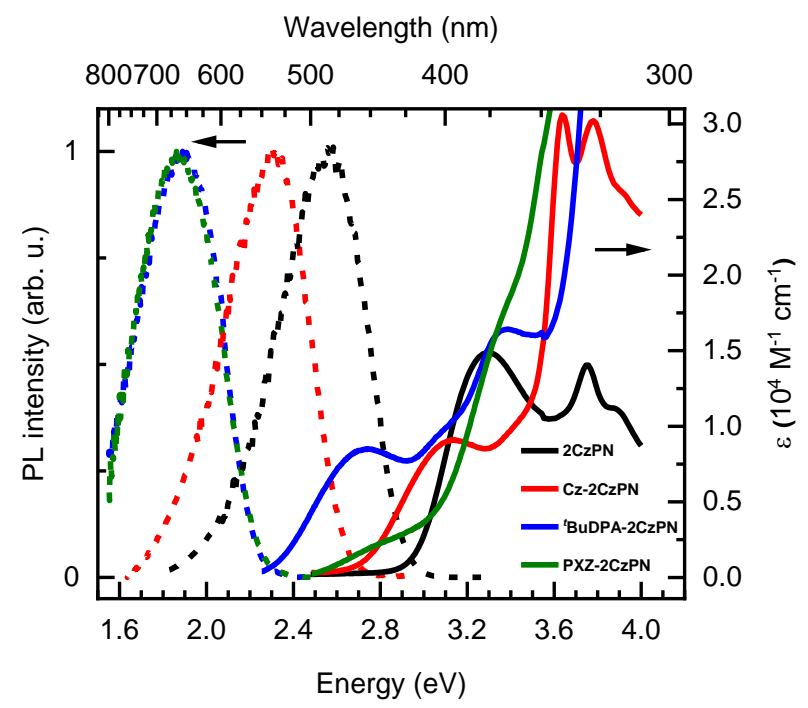

Figure 5. Absorption (solid line) and steady-state emission (dashed line) spectra of $2 \mathbf{C z P N}, \mathbf{C z}-\mathbf{2 C z P N}$,

${ }^{t}$ BuDPA-2CzPN, PXZ-2CzPN in toluene at $300 \mathrm{~K}\left(\lambda_{\mathrm{exc}}=300 \mathrm{~nm}\right)$.

All compounds show a broad, unstructured, low intensity absorption peak at the low energy side of the absorption band, with the peak positions indicated in Table 2. Along the series, the absorption maxima shift to lower energy, consistent with the trends observed for the HOMO-LUMO gap found from electrochemistry, and they reduce in intensity (Table 2). We assign these bands to a transition with a predominant $\mathrm{CT}$ character between the electron-rich donors and the electron-poor phthalonitrile, as predicted by Time-Dependent DFT calculations carried out within the Tamm-Dancoff approximation (TDA-DFT) calculations. The trend of the progressive decrease of the molar extinction coefficients of this low energy band reflects the increasing strength of the CT transition and is largely consistent with the trend in the theoretically calculated oscillator strengths (Table 2 ). The observation of extinction coefficients in the range of $10^{4} \mathrm{M}^{-1} \mathrm{~cm}^{-1}$ for the first three compounds in the series 
suggests the excited states involved have a mixed CT-LE character with a dominant CT character in agreement with the calculated electron-hole distributions shown in Figure $\mathbf{2}$ and the calculated $\varphi_{\mathrm{S}}$ listed in Table 2.

Table 2. Energetic positions and transition intensities for the $\mathrm{CT}$ and $\mathrm{S}_{1}$ state, derived from optical spectroscopy in toluene solution and from computational outputs at TDA-PBE0/6-31G(d,p).

\begin{tabular}{|c|c|c|c|c|c|c|}
\hline & $\begin{array}{l}\lambda_{\mathrm{abs}}(\mathrm{a}) \\
300 \mathrm{~K}\end{array}$ & $\begin{array}{c}\varepsilon_{\mathrm{CT}}{ }^{(\mathrm{b})} \\
\left(10^{4} \mathrm{M}^{-1} \mathrm{~cm}^{-1}\right)\end{array}$ & $\begin{array}{l}\lambda_{\mathrm{PL}}{ }^{(c)} \\
300 \mathrm{~K}\end{array}$ & $f^{(\mathrm{d})}$ & $S_{1}(e V)^{(e)}$ & $\varphi_{\mathrm{s}}{ }^{(\mathrm{f})}$ \\
\hline $2 \mathrm{CzPN}$ & $\begin{array}{l}3.30 \mathrm{eV} \\
(376 \mathrm{~nm})\end{array}$ & 1.49 & $\begin{array}{c}2.56 \mathrm{eV} \\
(484 \mathrm{~nm})\end{array}$ & 0.088 & 3.02 & 0.34 \\
\hline $\mathrm{Cz}-2 \mathrm{Cz} \mathrm{PN}$ & $\begin{array}{l}3.14 \mathrm{eV} \\
(395 \mathrm{~nm})\end{array}$ & 0.90 & $\begin{array}{c}2.32 \mathrm{eV} \\
(534 \mathrm{~nm})\end{array}$ & 0.023 & 2.33 & 0.20 \\
\hline${ }^{t}$ BuDPA-2CzPN & $\begin{array}{l}2.72 \mathrm{eV} \\
(456 \mathrm{~nm})\end{array}$ & 0.85 & $\begin{array}{c}1.88 \mathrm{eV} \\
(660 \mathrm{~nm})\end{array}$ & 0.047 & 2.18 & 0.28 \\
\hline PXZ-2CzPN & $\begin{array}{l}2.72 \mathrm{eV} \\
(456 \mathrm{~nm})\end{array}$ & 0.18 & $\begin{array}{l}1.88 \mathrm{eV} \\
(660 \mathrm{~nm})\end{array}$ & 0.003 & 1.67 & 0.11 \\
\hline
\end{tabular}

\footnotetext{
(a) Peak maximum of CT absorption.

(b) Molar extinction coefficient $\varepsilon_{\mathrm{cT}}$ for the compounds.

(c) Peak maximum of CT photoluminescence.

(d) Oscillator strength.

(e) Calculated vertical transition energy of $\mathrm{S}_{1}$.

(f) Indication of wavefunction overlap with a value of 1 implying a locally excited (LE) nature and a value of 0 suggesting a charge transfer (CT).
}

At higher energies $(E \geq 3.6 \mathrm{eV})$ in the absorption spectra, transitions localized on the conjugated donors are evident. For example, in the absorption spectrum of $\mathbf{2 C Z P N}$, there is a feature at $3.75 \mathrm{eV}$ (331 nm). We attribute this feature to a transition localized on the carbazole moiety since $\mathrm{N}$-phenyl- 
carbazole has an absorption peak at $3.65 \mathrm{eV},{ }^{34}$ and the shift to the blue side of the carbazole absorption in 2 CzPN by 10 meV will result from the acceptor unit withdrawing the electron density from the carbazole. Adding four further carbazole units to the compound to obtain $\mathbf{C z}-\mathbf{2} \mathbf{C z} \mathbf{P N}$, results in the appearance of an additional absorption peak at $3.65 \mathrm{eV}(340 \mathrm{~nm})$. We contend that the peak at 3.65 $\mathrm{eV}$ can be attributed to transitions localized on the inner carbazoles while the peak at $3.75 \mathrm{eV}$ results from those on the outer carbazoles, on the basis that the outer carbazoles donates some of their electron density to the inner carbazoles thus restoring the "normal" electron density of carbazoles there, while the outer carbazoles become electron deficient. In the case of ${ }^{t}$ BuDPA-2CZPN and PXZ2CZPN, there is a sharp increase in absorption with a peak at $4.10 \mathrm{eV}(302 \mathrm{~nm})$ and $3.88 \mathrm{eV}(320 \mathrm{~nm})$ (both off-scale in Figure 5), representing locally-excited (LE) transitions from diarylamine and phenoxazine ${ }^{35}$ units, respectively (see also Figure S10). The absorption band associated with the phthalonitrile acceptor is located at even higher energies with peaks from $4.6 \mathrm{eV}(270 \mathrm{~nm})$ onwards. ${ }^{36}$ Regarding the emission in toluene, all steady-state spectra are broad and structureless, characteristic of emission from a state with a dominant $\mathrm{CT}$ character. The photoluminescence maxima shift to lower energies across the family of derivatives, analogous to the shifts in the absorption maxima of the lowenergy $C T$ bands and in alignment with the TDA-DFT predicted $\mathrm{S}_{1}$ energies. $\Delta E_{S T}$ was determined from the measurements of fluorescence and phosphorescence spectra taken at the same temperature, namely $77 \mathrm{~K}$, to exclude different temperature-dependent energy shifts of the two emissions. This is shown in Figure $\mathbf{6 a}$, with the room temperature steady-state emission added for comparison. The steady-state emission at $77 \mathrm{~K}$ contains contributions from the prompt emission and delayed emission, which is mostly phosphorescence and, except for ${ }^{t}$ BuDPA-2CzPN, a negligible contribution of delayed fluorescence. For ${ }^{t}$ BuDPA-2CzPN, the delayed fluorescence was found to be still competing in intensity with phosphorescence at $77 \mathrm{~K}$, so that $\Delta E_{\mathrm{ST}}$ was determined from the $5 \mathrm{~K}$ measurements. The decrease in temperature leads to a blue-shift of the steady-state emission compared to its room-temperature spectrum. We attribute this to the freezing of the solvation shell at low temperatures that prevents reorganization of the solvent molecules after photoexcitation of the emitter molecules. ${ }^{37}$ To obtain the 
phosphorescence spectrum without contribution from fluorescence, we recorded the emission with detection in the millisecond range (see ESI for details). Table $\mathbf{3}$ below summarizes the energies obtained for the onsets of the singlet and triplet states along with the singlet-triplet gaps that are determined from them.

Table 3. Singlet and triplet energies in toluene solution and mCP film (10 wt\%).

\begin{tabular}{|c|c|c|c|c|c|c|}
\hline \multirow[b]{2}{*}{ Compound } & \multicolumn{3}{|c|}{ toluene } & \multicolumn{3}{|c|}{$\mathrm{mCP}$} \\
\hline & $S_{1}(e V)$ & $T_{1}(e V)$ & $\Delta E_{\mathrm{ST}}(\mathrm{eV})$ & $S_{1}(e V)$ & $\mathrm{T}_{1}(\mathrm{eV})$ & $\Delta E_{\mathrm{ST}}(\mathrm{eV})$ \\
\hline $2 \mathrm{CzPN}{ }^{(\mathrm{a})}$ & 3.03 & 2.73 & 0.30 & 2.85 & 2.62 & 0.23 \\
\hline $\mathrm{Cz}-2 \mathrm{Cz} P N^{(a)}$ & 2.85 & 2.69 & 0.16 & 2.69 & 2.62 & 0.07 \\
\hline${ }^{t} \mathrm{BuDPA}^{-2 C z P N}{ }^{(b)}$ & 2.37 & 2.36 & 0.01 & 2.34 & 2.33 & 0.01 \\
\hline PXZ-2CZPN ${ }^{\text {(a) }}$ & 2.57 & 2.56 & 0.01 & 2.40 & 2.37 & 0.03 \\
\hline
\end{tabular}

(a) Values determined using the onset at $77 \mathrm{~K}$.

(b) Values determined using the onset at $5 \mathrm{~K}$.

To address the CT or LE nature of the phosphorescence, we compared the position of the phosphorescence of the donor-acceptor compounds with the phosphorescence of the isolated donor and acceptor moieties themselves. For $\mathbf{2} \mathbf{C Z P N}$, the phosphorescence maximum is much lower in energy than both the phosphorescence peak of carbazole and of phthalonitrile [with peaks at $2.64 \mathrm{eV} v s$ $3.0 \mathrm{eV}^{34}$ and $3.2 \mathrm{eV}^{38},(470 \mathrm{~nm}$ vs $410 \mathrm{~nm}$ and $390 \mathrm{~nm})$ respectively]. Thus, the spectroscopic data clearly identify the phosphorescence in 2CzPN as a predominant CT transition with some LE contributions, as evident from the vibrational structure. This is in agreement and supported by the quantum chemical calculations (Figure $\mathbf{S 2}$, Table $\mathbf{S 2}$ ) and the work by Wong et $\mathrm{al.}{ }^{20}$ The same reasoning assigns the phosphorescence in the three derivatives as predominantly $\mathrm{CT}$ transitions. The $\Delta E_{\mathrm{ST}}$ values in solution decrease along the series from $0.30 \mathrm{eV}$ for the parent compound $2 \mathrm{CzPN}$ to $0.01 \mathrm{eV}$ for ${ }^{t}$ BuDPA-2CZPN and PXZ-2CZPN. This decrease reflects the increasing hole localization on the donor dendron, which is consistent with the DFT calculations. Thus, the concept underlying the chemical design is confirmed. 

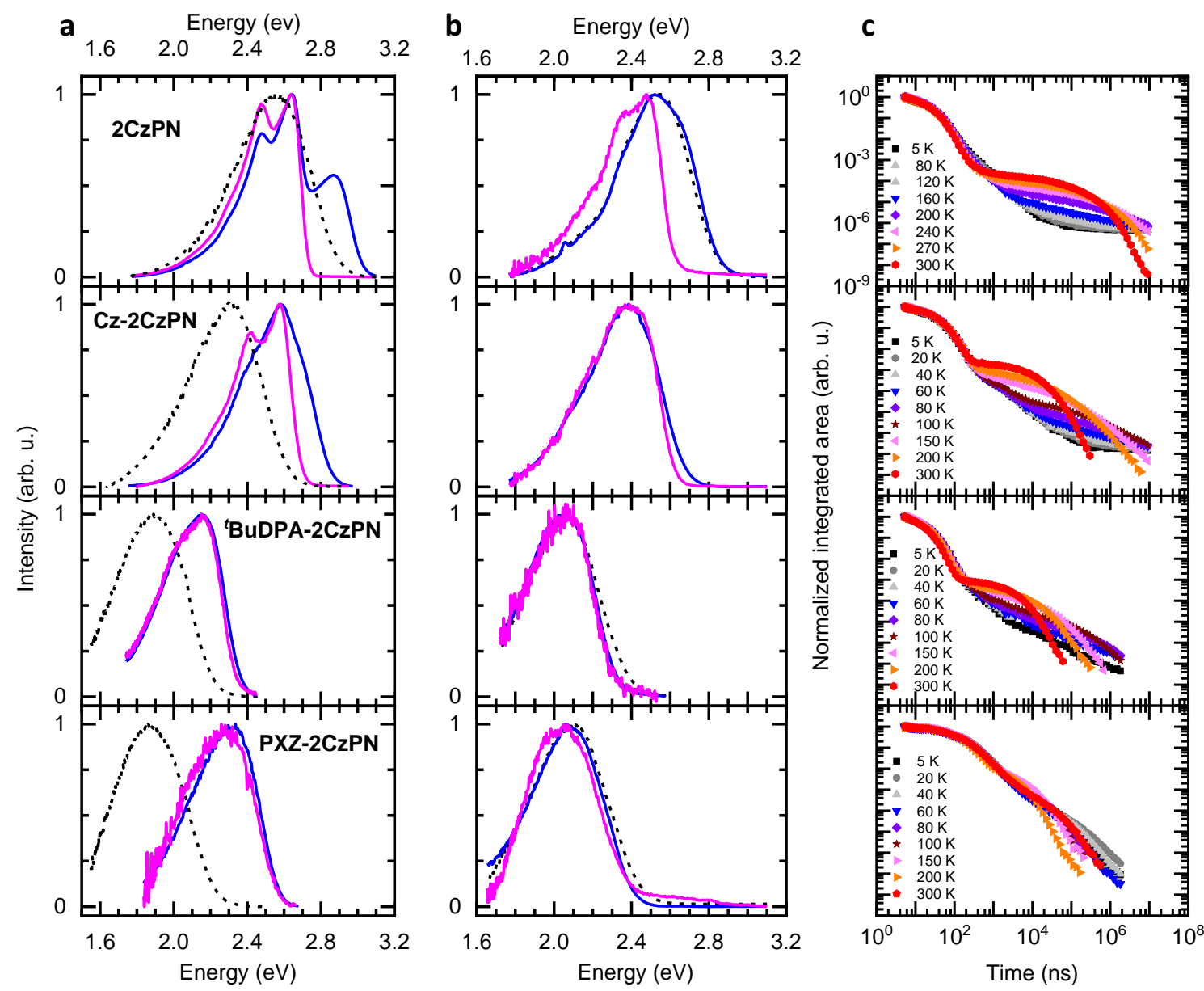

Figure 6. PL emission and transient decay of $2 \mathrm{Cz} P N, \mathrm{Cz}-2 \mathrm{CzPN},{ }^{t}$ BuDPA-2CzPN, PXZ-2CzPN. Black dashed lines denote the steady-state PL at $300 \mathrm{~K}$. Blue lines denote the steady-state emission at $77 \mathrm{~K}$. Magenta lines denote the PL with detection in the ms range (see ESI) at $77 \mathrm{~K}^{*}$. (a) PL emission in toluene for excitation at the maximum of the CT absorption band. (b) PL emission in $10 \mathrm{wt} \% \mathrm{mCP}$ film $\left(\lambda_{\text {exc }}=300 \mathrm{~nm}\right) .(\mathrm{c})$ Transient PL decay in $10 \mathrm{wt} \% \mathrm{mCP}$ film at $300 \mathrm{~K}\left(\lambda_{\mathrm{exc}}=355 \mathrm{~nm}\right) .{ }^{*}$ For ${ }^{t}$ BuDPA-2CzPN, the steady-state emission and PL with the detection in the ms range is obtained at $5 \mathrm{~K}\left(\lambda_{\mathrm{exc}}=355 \mathrm{~nm}\right)$. 
To understand how the photophysical properties in solution transfer into film, we next investigated the compounds in $10 \mathrm{wt} \% \mathrm{mCP}$ film $[\mathrm{mCP}=1,3$-bis $(N$-carbazolyl $)$ benzene $]$, where $\mathrm{mCP}$ is a host matrix with sufficiently high triplet energy $(2.90 \mathrm{eV})$ to ensure confinement of the triplet excitons on the emitter. ${ }^{39}$ Figure $\mathbf{6 b}$ shows the thin film PL emission for the series at $300 \mathrm{~K}$ and $77 \mathrm{~K}$ ( $5 \mathrm{~K}$ for ${ }^{t}$ BuDPA2CZPN). We find the same trend in the singlet-triplet gap as in solution with $\Delta E_{S \mathrm{~T}}$ decreasing along the series (within the experimental error margin of $10 \mathrm{meV}$, Table 3). In contrast to solution, the roomtemperature steady-state spectra almost coincide with the low-temperature steady-state spectra. We attribute this to the absence of solvent reorganization after excitation in the solid matrix. ${ }^{37}$ It implies that the spectra and energy gaps obtained at low-temperature represent the room temperature situation well.

For pronounced TADF, a small $\Delta E_{\mathrm{ST}}$ value is a necessary but not a sufficient condition. To evaluate the potential of a molecule as a TADF emitter, we also need to consider the relevant rates of radiative and non-radiative decay, as reflected in the PLQY, delayed emission lifetimes, and the contribution of the TADF to the total emission. With a view to their use as emitters in OLEDs, we focus the discussion on the TADF-related parameters in film, comparing with the solution data wherever relevant. Figure $\mathbf{6 c}$ shows the transient PL decays for the compounds in $10 \mathrm{wt} \% \mathrm{mCP}$ film. The analogous data for solution are given in the ESI, along with a complete list of parameters used in the mono-exponential fittings for transient decay profiles in solution and film (Figure S11, Table S7). The photophysical parameters obtained are summarized in Table $\mathbf{4}$ for solution and Table $\mathbf{5}$ for films.

For both solution and film, we can identify two regimes in the PL decay, that is, a prompt decay in the nanosecond range and a delayed emission that takes place in the microsecond range. We can safely attribute the delayed emission to TADF, based on the small $\Delta E_{S T}$ gap and the increase of the emission in the microsecond range with increasing temperature. From the temperature dependence of the 
delayed fluorescence (DF) in the film we derive the activation energy for TADF (Table 5, Figure S12). As with the singlet-triplet gap, the activation energy also decreases along the series. The lower or comparable activation energy than the $\Delta E_{S T}$ measured from the steady-state emission spectra together with the prediction from TDA-DFT calculations of closely-lying $T_{1}, T_{2}$ and $S_{1}$ point to the involvement of higher-lying triplet states (Table S2). We note that the difference between $\Delta E_{S T}$ and the measured activation energy is strongest for $2 \mathrm{CzPN}$, which is the compound with the highest LE contribution in $\mathrm{T}_{2}$ which is expected to lead to the highest spin-orbit coupling with the $\mathrm{S}_{1} \mathrm{CT}$ state.

Table 4. Quantum yields $\Phi$, lifetimes $\tau$ and rates $k$ for the compounds in toluene.

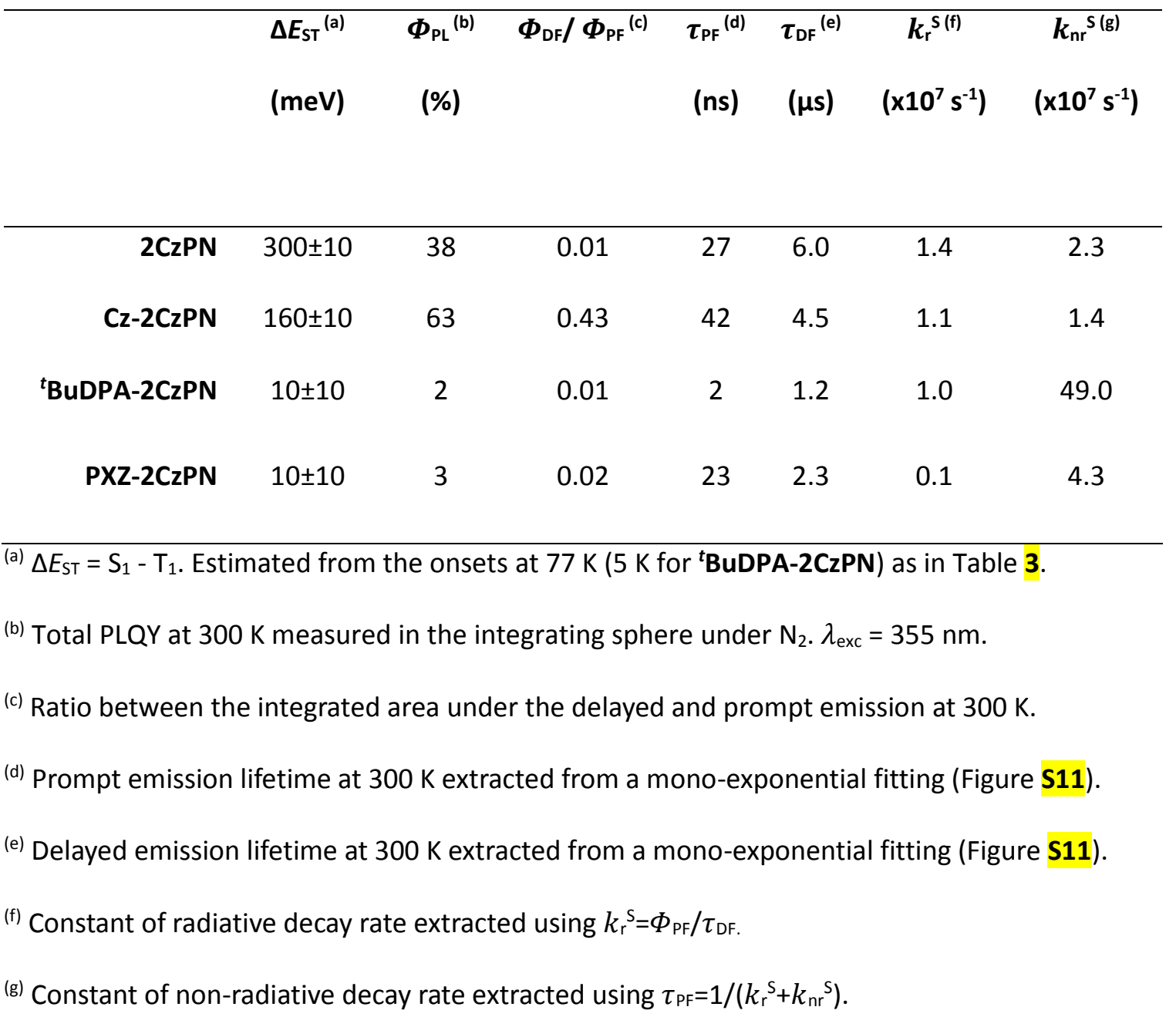


Table 5. Quantum yields $\Phi$, activation energies $E_{\text {act, }}$ lifetimes $\tau$ and rates $k$ for the compounds in $\mathrm{mCP}$ films.

\begin{tabular}{|c|c|c|c|c|c|c|c|c|c|c|}
\hline & \multirow{2}{*}{$\begin{array}{l}\Delta E_{S \mathrm{~T}}{ }^{\text {(a) }} \\
(\mathrm{meV})\end{array}$} & \multirow{2}{*}{$\begin{array}{c}E_{\text {act }} \\
(\mathrm{meV})\end{array}$} & \multirow{2}{*}{$\begin{array}{c}\Phi_{\mathrm{PL}}{ }^{(\mathbf{b})} \\
(\%)\end{array}$} & \multirow[t]{2}{*}{$\Phi_{\mathrm{DF}} / \Phi_{\mathrm{PF}}{ }^{(c)}$} & \multirow{2}{*}{$\begin{array}{c}\tau_{\mathrm{PF}}{ }^{(\mathrm{d})} \\
\text { (ns) }\end{array}$} & \multirow{2}{*}{$\begin{array}{c}\tau_{\mathrm{DF}}(\mathrm{e}) \\
(\mu \mathrm{s})\end{array}$} & \multirow{2}{*}{$\begin{array}{l}k_{r}^{S(f)} \\
\left(\times 10^{7}\right.\end{array}$} & \multirow{2}{*}{$\begin{array}{l}k_{\mathrm{nr}}{ }^{\mathrm{s}(\mathrm{g})} \\
\left(\times 10^{7}\right.\end{array}$} & \multicolumn{2}{|c|}{$\tau_{\mathrm{Ph}}{ }^{(\mathrm{h})}$} \\
\hline & & & & & & & & & (ms) & (ms) \\
\hline & & & & & & & $\left.s^{-1}\right)$ & $\left.s^{-1}\right)$ & $5 \mathrm{~K}$ & $80 \mathrm{~K}$ \\
\hline 2CzPN & $230 \pm 10$ & $75 \pm 5$ & 93 & 1.0 & 16 & 42.6 & 2.9 & 3.4 & 170 & 110 \\
\hline $\mathrm{Cz}-2 \mathrm{CzPN}$ & $70 \pm 10$ & $39 \pm 3$ & 78 & 0.6 & 25 & 7.0 & 1.9 & 2.1 & 150 & 60 \\
\hline${ }^{t}$ BuDPA-2CzPN & $10 \pm 10$ & $21 \pm 1$ & 14 & 0.1 & 10 & 2.3 & 1.2 & 8.8 & 3.5 & - \\
\hline PXZ-2CZPN & $30 \pm 10$ & - & 23 & 0.3 & 95 & 2.5 & 0.2 & 0.9 & 0.2 & - \\
\hline
\end{tabular}

(a) $\Delta E_{S T}=\mathrm{S}_{1}-\mathrm{T}_{1}$. Estimated from the onsets as in Table $\mathbf{3}$.

(b) Total PLQY at $300 \mathrm{~K}$ measured in the integrating sphere under $\mathrm{N}_{2} \cdot \lambda_{\mathrm{exc}}=355 \mathrm{~nm}$.

${ }^{(c)}$ Ratio between the integrated area under the delayed and prompt emission.

(d) Prompt emission lifetime at $300 \mathrm{~K}$ extracted from a mono-exponential fitting (Figure S11).

(e) Delayed emission lifetime at $300 \mathrm{~K}$ extracted from a mono-exponential fitting (Figure S11).

${ }^{(f)}$ Constant of radiative decay rate extracted using $k_{\mathrm{r}}^{\mathrm{S}}=\Phi_{\mathrm{PF}} / \tau_{\mathrm{DF}}$.

${ }^{(\mathrm{g})}$ Constant of non-radiative decay rate extracted using $\tau_{\mathrm{PF}}=1 /\left(k_{\mathrm{r}}^{\mathrm{S}}+k_{\mathrm{nr}}{ }^{\mathrm{S}}\right)$.

(h) Phosphorescence lifetime extracted from a mono-exponential fitting (Figure S13).

We note that for $\mathrm{Cz}-2 \mathrm{CzPN}$ and ${ }^{t} \mathrm{BuDPA}-\mathbf{2 C z P N}$ the radiative decay rate from the singlet state $k_{\mathrm{r}}{ }^{\mathrm{s}}$ is only slightly reduced relative to the parent compound, while it is significantly reduced for PXZ-2CZPN, indicating a too strong decoupling of the hole and electron wavefunctions for this compound. This is consistent with both the low calculated oscillator strength and the low PLQY in PXZ-2CZPN. For ${ }^{t}$ BuDPA-2CZPN, the $\Delta E_{S T}$ is small and a high radiative rate from the CT singlet is maintained, yet a high non-radiative CT singlet decay rate precludes a high photoluminescence quantum yield. This finding is consistent with the work of Yang et al. ${ }^{40-41}$ where they reported a diphenylsulfone core linked to acridine-based donor dendrons. When they attached diphenylamine or carbazole as peripheral donor 
groups they also found a smaller gap and lower PLQY in toluene for the former ( $40 \mathrm{meV}, 12 \%$ ) than for the latter $(90 \mathrm{meV}, 68 \%) .^{40-41}$

Despite the small $\Delta E_{S T}$, we observe very little TADF in solution except for Cz-2CzPN (c.f. column 3 in Table 4, Table S8). A low TADF yield in solution at room temperature has been observed previously in compounds otherwise predicted to show TADF. ${ }^{20,42}$ We attribute this to enhanced quenching of the triplet state in solution compared to film, which can occur, for example, by collisions with solvent molecules. The fact that quenching does not occur for $\mathbf{C z - 2 C z P N}$ hints that its triplet may be localized somewhat more on the inner carbazole of the donor dendrons, where it is more shielded from intermolecular quenching. Thus, in solution, the emission characteristics are dominated by the prompt singlet state emission.

In the film, the rigid matrix removes this collisional quenching mechanism, so that TADF has a stronger contribution to the overall PLQY. Nevertheless, the ratio between the integrated emission intensities of delayed and prompt fluorescence is less than 4 for all compounds, including the parent $2 \mathrm{CzPN}$, so that the commonly used approximation forwarded by Dias et al. to calculate the RISC rate does not apply. ${ }^{43} \mathrm{An}$ assessment of the efficiency of RISC is still possible by considering the DF/PF ratio (PF = prompt fluorescence) and the DF lifetime together. As summarized in Table $\mathbf{5}$, the three $\mathbf{2 C z P N}$ derivatives show a shorter DF lifetime than the parent compound. While this by itself could indicate a faster RISC rate, the lower DF/PF ratios in the extended compounds indicate that there must be an additional dominant process present. We conclude that the shorter lifetime of the DF is thus rather associated with a quenching channel for the triplet states from which the DF is fed. We confirmed this by measuring the phosphorescence lifetimes. The triplet lifetime of ${ }^{\mathrm{t}} \mathrm{BuDPA}-2 \mathrm{Cz} P N$ and PXZ-2CzPN at $5 \mathrm{~K}$ is about 50 and 1000 times shorter than that for the parent $2 \mathrm{CzPN}$. For $\mathbf{C z}-\mathbf{2 C z P N}$, it is comparable with $2 \mathrm{Cz} \mathbf{P N}$ at $5 \mathrm{~K}$, yet decreases more strongly upon heating, for example, to $80 \mathrm{~K}$, thus also indicating 
thermally activated non-radiative decay channels (Table 5, and Figure S13). The nearly identical triplet energy of 2 CzPN and $\mathbf{C z}-\mathbf{2 C z P N}$ excludes the energy-gap law as the origin. Most likely, the shorter triplet lifetime results from the higher conformational degrees of freedom in the three $\mathbf{2 C Z P N}$ derivatives, so that deactivation by torsional modes is facilitated. Overall, we find that employing donor dendrons indeed reduces the singlet-triplet gap, and concomitantly the activation energy for TADF. However, in the film this advantage is compromised by an increased non-radiative decay from the triplet state, which occurs in different degrees for the different extensions.

\section{Concluding discussion}

While employing donor dendrons has been explored before as a means to increase the TADF yield, often with the same donor units used throughout the dendron, there are few investigations into the relationship between the nature of the donor dendron and the reduction in the singlet-triplet gap. ${ }^{44}$ In our work, we synthesized a series of molecules based on the TADF-active 2CzPN where we increased the hole-localization through suitable decoration of the carbazole donors. Our results show that the singlet-triplet gap can be reduced while maintaining a sufficiently high oscillator strength for efficient emission. Indeed, $\mathbf{C z - 2 C z P N}$ demonstrates the most suitable balance in charge distribution where in solution this compound significantly exceeds the PLQY and TADF properties of the parent 2CzPN. However, we also find that, in the film, a high non-radiative rate of the triplet state prohibits a stronger role of TADF in the extended donor molecules despite a reduced gap between the singlet and triplet state. Our results imply that the approach of fine-tuning the donor dendron strength indeed can improve the TADF emission, yet for implementation, this still requires more control over the nonradiative decay of the triplet state in the film by, for instance, ensuring that the donor dendrons are sufficiently rigid. 


\section{Methods}

We prepared solutions of $0.05 \mathrm{mg} / \mathrm{mL}$ in toluene (corresponding to $10^{-4}-10^{-5} \mathrm{M}$ depending on molecular weight) as well as spin-coated $10 \mathrm{wt} \%$ films in $\mathrm{mCP}$ and measured them at different temperatures in steady-state mode using a Jasco FP-8600 spectrofluorimeter. In time-resolved mode they were measured using an iCCD camera (Andor iStar A-DH334T-18F-03) by exponentially increasing delay and gating times where the gating time is kept lower by 10 times compared to the delay time. For the steady-state mode, variable excitation wavelengths were used (see figure captions) while for the iCCD measurements samples were excited at $355 \mathrm{~nm}$. The quantum chemical calculations were performed using the Gaussian 09 (revision D.0184) suite for the DFT. ${ }^{45}$ Molecular structures were optimised in the ground state geometry using $\mathrm{PBEO}^{46}$ functionals each employing the $6-31 \mathrm{G}(\mathrm{d}, \mathrm{p})$ basis set with dispersion effects included. ${ }^{47}$ Excited state calculations were performed within the Tamn-Dancoff approximation (TDA) at the same level of theory. ${ }^{48}$ Full experimental and computational details as well as the information on the synthesis and chemical characterization are provided in the ESI.

\section{Acknowledgements}

This project has received funding from the European Union's Horizon 2020 research and innovation programme under the Marie Skłodowska-Curie grant agreement No 812872 (TADFlife). SB acknowledges support from the German Science Foundation (392306670/HU2362). The St Andrews team would also like to thank the Leverhulme Trust (RPG-2016-047) and EPSRC (EP/P010482/1) for financial support. Computational resources have been provided by the Consortium des Équipements de Calcul Intensif (CÉCl), funded by the Fonds de la Recherche Scientifiques de Belgique (F.R.S.-FNRS) under Grant No. 2.5020.11. DB is a FNRS Research Director. Y.O. acknowledges funding from the FRSFNRS under the grant F.4534.21 (MIS-IMAGINE). Y.O. is grateful for the fruitful discussions with Prof. Juan-Carlos Sancho-Garcia from the University of Alicante and Prof. Luca Muccioli from the University of Bologna. 


\section{References}

1. Wong, M. Y.; Zysman-Colman, E., Purely Organic Thermally Activated Delayed Fluorescence Materials for Organic Light-Emitting Diodes. Adv. Mater. 2017, 29, 1605444.

2. Godumala, M.; Choi, S.; Cho, M. J.; Choi, D. H., Recent breakthroughs in thermally activated delayed fluorescence organic light emitting diodes containing non-doped emitting layers. J. Mater. Chem. C 2019, 7, 2172-2198.

3. Zou, S.-J.; Shen, Y.; Xie, F.-M.; Chen, J.-D.; Li, Y.-Q.; Tang, J.-X., Recent advances in organic lightemitting diodes: toward smart lighting and displays. Mater. Chem. Front. 2020, 4, 788-820.

4. Gibson, J.; Penfold, T. J., Nonadiabatic coupling reduces the activation energy in thermally activated delayed fluorescence. Phys. Chem. Chem. Phys. 2017, 19, 8428-8434.

5. Miwa, T.; Kubo, S.; Shizu, K.; Komino, T.; Adachi, C.; Kaji, H., Blue organic light-emitting diodes realizing external quantum efficiency over $25 \%$ using thermally activated delayed fluorescence emitters. Sci. Rep. 2017, 7, 284.

6. Kaji, H.; Suzuki, H.; Fukushima, T.; Shizu, K.; Suzuki, K.; Kubo, S.; Komino, T.; Oiwa, H.; Suzuki, F.; Wakamiya, A.; Murata, Y.; Adachi, C., Purely organic electroluminescent material realizing $100 \%$ conversion from electricity to light. Nat. Commun. 2015, 6, 8476.

7. Shizu, K.; Tanaka, H.; Uejima, M.; Sato, T.; Tanaka, K.; Kaji, H.; Adachi, C., Strategy for Designing Electron Donors for Thermally Activated Delayed Fluorescence Emitters. J. Phys. Chem. C 2015, 119, 1291-1297.

8. Stachelek, P.; Ward, J. S.; dos Santos, P. L.; Danos, A.; Colella, M.; Haase, N.; Raynes, S. J.; Batsanov, A. S.; Bryce, M. R.; Monkman, A. P., Molecular Design Strategies for Color Tuning of Blue TADF Emitters. ACS Appl. Mater. Interfaces 2019, 11, 27125-27133.

9. Rajamalli, P.; Chen, D.; Li, W.; Samuel, I. D. W.; Cordes, D. B.; Slawin, A. M. Z.; Zysman-Colman, E., Enhanced thermally activated delayed fluorescence through bridge modification in sulfone-based emitters employed in deep blue organic light-emitting diodes. J. Mater. Chem. C 2019, 7, 6664-6671. 
10. Li, J.; Liao, X.; Xu, H.; Li, L.; Zhang, J.; Wang, H.; Xu, B., Deep-blue thermally activated delayed fluorescence dendrimers with reduced singlet-triplet energy gap for low roll-off non-doped solutionprocessed organic light-emitting diodes. Dyes Pigm. 2017, 140, 79-86.

11. Matsuoka, K.; Albrecht, K.; Yamamoto, K.; Fujita, K., Mulifunctional Dendritic Emitter: Aggregation-Induced Emission Enhanced, Thermally Activated Delayed Fluorescent Material for Solution-Processed Multilayered Organic Light-Emitting Diodes. Sci. Rep. 2017, 7, 41780.

12. Albrecht, K.; Matsuoka, K.; Yokoyama, D.; Sakai, Y.; Nakayama, A.; Fujita, K.; Yamamoto, K., Thermally activated delayed fluorescence OLEDs with fully solution processed organic layers exhibiting nearly 10\% external quantum efficiency. Chem. Commun. 2017, 53, 2439-2442.

13. Huang, M.; Li, Y.; Wu, K.; Luo, J.; Xie, G.; Li, L.; Yang, C., Carbazole-dendronized thermally activated delayed fluorescent molecules with small singlet-triplet gaps for solution-processed organic light-emitting diodes. Dyes Pigm. 2018, 153, 92-98.

14. Li, Y.; Xie, G.; Gong, S.; Wu, K.; Yang, C., Dendronized delayed fluorescence emitters for nondoped, solution-processed organic light-emitting diodes with high efficiency and low efficiency roll-off simultaneously: two parallel emissive channels. Chem. Sci. 2016, 7, 5441-5447.

15. Kotadiya, N. B.; Blom, P. W. M.; Wetzelaer, G.-J. A. H., Efficient and stable single-layer organic light-emitting diodes based on thermally activated delayed fluorescence. Nat. Photonics 2019, 13, 765769.

16. Chatterjee, T.; Wong, K.-T., Perspective on Host Materials for Thermally Activated Delayed Fluorescence Organic Light Emitting Diodes. Adv. Opt. Mater. 2019, 7, 1800565.

17. Rodella, F.; Bagnich, S.; Duda, E.; Meier, T.; Kahle, J.; Athanasopoulos, S.; Köhler, A.; Strohriegl, P., High Triplet Energy Host Materials for Blue TADF OLEDs-A Tool Box Approach. Front. Chem. 2020, 8,657 .

18. Uoyama, H.; Goushi, K.; Shizu, K.; Nomura, H.; Adachi, C., Highly efficient organic light-emitting diodes from delayed fluorescence. Nature 2012, 492, 234-238. 
19. Kim, G. H.; Lampande, R.; Im, J. B.; Lee, J. M.; Lee, J. Y.; Kwon, J. H., Controlling the exciton lifetime of blue thermally activated delayed fluorescence emitters using a heteroatom-containing pyridoindole donor moiety. Mater. Horiz. 2017, 4, 619-624.

20. Wong, M. Y.; Krotkus, S.; Copley, G.; Li, W.; Murawski, C.; Hall, D.; Hedley, G. J.; Jaricot, M.; Cordes, D. B.; Slawin, A. M. Z.; Olivier, Y.; Beljonne, D.; Muccioli, L.; Moral, M.; Sancho-Garcia, J.-C.; Gather, M. C.; Samuel, I. D. W.; Zysman-Colman, E., Deep-Blue Oxadiazole-Containing Thermally Activated Delayed Fluorescence Emitters for Organic Light-Emitting Diodes. ACS Appl. Mater. Interfaces 2018, 10, 33360-33372.

21. Dreuw, A.; Head-Gordon, M., Single-Reference ab Initio Methods for the Calculation of Excited States of Large Molecules. Chem. Rev. 2005, 105, 4009-4037.

22. Etienne, T.; Assfeld, X.; Monari, A., New Insight into the Topology of Excited States through Detachment/Attachment Density Matrices-Based Centroids of Charge. J. Chem. Theory Comput. 2014, 10, 3906-3914.

23. Noda, H.; Nakanotani, H.; Adachi, C., Excited state engineering for efficient reverse intersystem crossing. Sci. Adv. 2018, 4, eaao6910.

24. Noda, H.; Chen, X.-K.; Nakanotani, H.; Hosokai, T.; Miyajima, M.; Notsuka, N.; Kashima, Y.; Brédas, J.-L.; Adachi, C., Critical role of intermediate electronic states for spin-flip processes in chargetransfer-type organic molecules with multiple donors and acceptors. Nat. Mater. 2019, 18, 1084-1090. 25. Zhao, Y.; Truhlar, D. G., The M06 suite of density functionals for main group thermochemistry, thermochemical kinetics, noncovalent interactions, excited states, and transition elements: two new functionals and systematic testing of four M06-class functionals and 12 other functionals. Theor. Chem. Acc. 2007, 120, 215-241.

26. Vydrov, O. A.; Scuseria, G. E., Assessment of a long-range corrected hybrid functional. J. Chem. Phys. 2006, 125, 234109. 
27. Olivier, Y.; Yurash, B.; Muccioli, L.; D’Avino, G.; Mikhnenko, O.; Sancho-García, J. C.; Adachi, C.; Nguyen, T. Q.; Beljonne, D., Nature of the singlet and triplet excitations mediating thermally activated delayed fluorescence. Phys. Rev. Mater. 2017, 1, 075602.

28. Etherington, M. K.; Franchello, F.; Gibson, J.; Northey, T.; Santos, J.; Ward, J. S.; Higginbotham, H. F.; Data, P.; Kurowska, A.; Dos Santos, P. L.; Graves, D. R.; Batsanov, A. S.; Dias, F. B.; Bryce, M. R.; Penfold, T. J.; Monkman, A. P., Regio- and conformational isomerization critical to design of efficient thermally-activated delayed fluorescence emitters. Nat. Commun. 2017, 8, 14987.

29. Karon, K.; Lapkowski, M., Carbazole electrochemistry: a short review. J. Solid State Electrochem. 2015, 19, 2601-2610.

30. Ambrose, J. F.; Nelson, R. F., Anodic Oxidation Pathways of Carbazoles: I. Carbazole and NSubstituted Derivatives. J. Electrochem. Soc. 1968, 115, 1159.

31. Karon, K.; Lapkowski, M.; Juozas, G., Electrochemical and UV-Vis/ESR spectroelectrochemical properties of polymers obtained from isomeric 2,7- and 3,6- linked carbazole trimers; influence of the linking topology on polymers properties. Electrochim. Acta 2014, 123, 176-182.

32. Connelly, N. G.; Geiger, W. E., Chemical Redox Agents for Organometallic Chemistry. Chem. Rev. 1996, 96, 877-910.

33. Cardona, C. M.; Li, W.; Kaifer, A. E.; Stockdale, D.; Bazan, G. C., Electrochemical Considerations for Determining Absolute Frontier Orbital Energy Levels of Conjugated Polymers for Solar Cell Applications. Adv. Mater. 2011, 23, 2367-2371.

34. Bagnich, S. A.; Athanasopoulos, S.; Rudnick, A.; Schroegel, P.; Bauer, I.; Greenham, N. C.; Strohriegl, P.; Köhler, A., Excimer Formation by Steric Twisting in Carbazole and Triphenylamine-Based Host Materials. J. Phys. Chem. C 2015, 119, 2380-2387.

35. Mantsch, H. H.; Dehler, J., $\pi$-Electronic structure and reactivity of phenoxazine (1), phenothiazine (2), and phenoxthiin (3). Can. J. Chem. 1969, 47, 3173-3178.

36. Takei, K.; Kanda, Y., Phosphorescence spectra of benzonitrile and related compounds. Spectrochim. Acta 1962, 18, 1201-1216. 
37. Lakowicz, J. R., Principles of Fluorescence Spectroscopy. 3rd ed.; Springer: Berlin, 2006.

38. Hayashi, H.; Nagakura, S., The E.S.R. and phosphorescence spectra of some dicyanobenzene complexes with methyl-substituted benzenes. Mol. Phys. 1970, 19, 45-53.

39. Ren, X.; Li, J.; Holmes, R. J.; Djurovich, P. I.; Forrest, S. R.; Thompson, M. E., Ultrahigh Energy Gap Hosts in Deep Blue Organic Electrophosphorescent Devices. Chem. Mater. 2004, 16, 4743-4747.

40. Gong, S.; Luo, J.; Wang, Z.; Li, Y.; Chen, T.; Xie, G.; Yang, C., Tuning emissive characteristics and singlet-triplet energy splitting of fluorescent emitters by encapsulation group modification: Yellow TADF emitter for solution-processed OLEDs with high luminance and ultraslow efficiency roll-off. Dyes Pigm. 2017, 139, 593-600.

41. Luo, J.; Gong, S.; Gu, Y.; Chen, T.; Li, Y.; Zhong, C.; Xie, G.; Yang, C., Multi-carbazole encapsulation as a simple strategy for the construction of solution-processed, non-doped thermally activated delayed fluorescence emitters. J. Mater. Chem. C 2016, 4, 2442-2446.

42. Lee, J.; Shizu, K.; Tanaka, H.; Nakanotani, H.; Yasuda, T.; Kaji, H.; Adachi, C., Controlled emission colors and singlet-triplet energy gaps of dihydrophenazine-based thermally activated delayed fluorescence emitters. J. Mater. Chem. C 2015, 3, 2175-2181.

43. Dias, F. B.; Penfold, T. J.; Monkman, A. P., Photophysics of thermally activated delayed fluorescence molecules. Method. Appl. Fluoresc. 2017, 5, 012001.

44. Park, W. J.; Lee, Y.; Kim, J. Y.; Yoon, D. W.; Kim, J.; Chae, S. H.; Kim, H.; Lee, G.; Shim, S.; Yang, J. H.; Lee, S. J., Effective thermally activated delayed fluorescence emitter and its performance in OLED device. Synth. Met. 2015, 209, 99-104.

45. M. J. Frisch, G. W. T., H. B. Schlegel, G. E. Scuseria, M. A. Robb, J. R. Cheeseman, G. Scalmani, V. Barone, B. Mennucci, G. A. Petersson, H. Nakatsuji, M. Caricato, X. Li, H. P. Hratchian, A. F. Izmaylov, J. Bloino, G. Zheng, J. L. Sonnenberg, M. Hada, M. Ehara, K. Toyota, R. Fukuda, J. Hasegawa, M. Ishida, T. Nakajima, Y. Honda, O. Kitao, H. Nakai, T. Vreven, J. A. Montgomery Jr., J. E. Peralta, F. Ogliaro, M. Bearpark, J. J. Heyd, E. Brothers, K. N. Kudin, V. N. Staroverov, R. Kobayashi, J. Normand, K. Raghavachari, A. Rendell, J. C. Burant, S. S. Iyengar, J. Tomasi, M. Cossi, N. Rega, J. M. Millam, M. Klene, 
J. E. Knox, J. B. Cross, V. Bakken, C. Adamo, J. Jaramillo, R. Gomperts, R. E. Stratmann, O. Yazyev, A. J. Austin, R. Cammi, C. Pomelli, J. W. Ochterski, R. L. Martin, K. Morokuma, V. G. Zakrzewski, G. A. Voth, P. Salvador, J. J. Dannenberg, S. Dapprich, A. D. Daniels, Ö. Farkas, J. B. Foresman, J. V. Ortiz, J. Cioslowski, and D. J. Fox, Gaussian, Inc., Wallingford CT, 2013.

46. Adamo, C.; Barone, V., Toward reliable density functional methods without adjustable parameters: The PBE0 model. J. Chem. Phys. 1999, 110, 6158-6170.

47. Dunning, T. H., Gaussian basis sets for use in correlated molecular calculations. I. The atoms boron through neon and hydrogen. J. Chem. Phys. 1989, 90, 1007-1023.

48. Hirata, S.; Head-Gordon, M., Time-dependent density functional theory within the TammDancoff approximation. Chem. Phys. Lett. 1999, 314, 291-299. 
For Table of Contents Only:

Fine-tuning hole-electron overlap for TADF

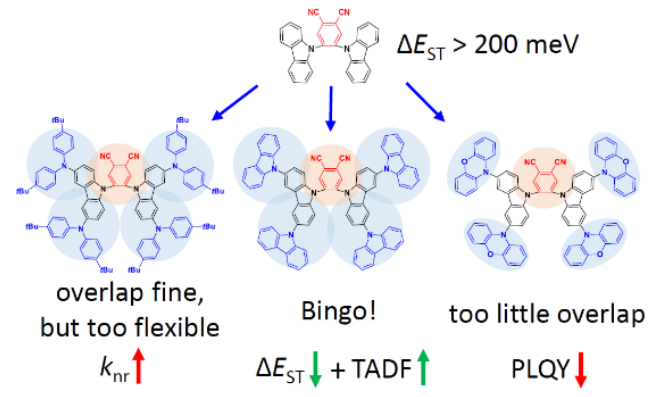

